\title{
REMOÇÃO DOS HORMÔNIOS 17ß-ESTRADIOL E 17 $\alpha$-ETINILESTRADIOL DE SOLUÇÕES AQUOSAS EMPREGANDO TURFA DECOMPOSTA COMO MATERIAL ADSORVENTE
}

\author{
Andreia N. Fernandes*,\# ${ }^{*}$ Marcelo Giovanela \\ Centro de Ciências Exatas e Tecnologia, Universidade de Caxias do Sul, 95070-560 Caxias do Sul - RS, Brasil \\ Carlos A. P. Almeida \\ Departamento de Química, Universidade do Estadual do Centro Oeste, 85040-080 Guarapuava - PR, Brasil \\ Valdemar I. Esteves \\ Centro de Estudos do Ambiente e do Mar e Departamento de Química, Universidade de Aveiro, 3810-193, Aveiro, Portugal \\ Maria M. D. Sierra \\ Departamento de Química, Universidade Federal de Santa Catarina, 88040-900 Florianópolis - SC, Brasil \\ Marco T. Grassi \\ Departamento de Química, Universidade Federal do Paraná, 81531-990 Paraná - PR, Brasil
}

Recebido em 9/11/10; aceito em 14/4/11; publicado na web em 10/6/11

\begin{abstract}
REMOVAL OF THE HORMONES 17 $\beta$-ESTRADIOL AND 17 $\alpha$-ETHINYLESTRADIOL FROM AQUEOUS SOLUTIONS EMPLOYING A DECOMPOSED PEAT AS ADSORBENT MATERIAL. This paper describes the adsorption of 17 $\beta$-estradiol (E2) and $17 \alpha$-ethinylestradiol (EE2) from aqueous solution by decomposed peat. The peat presented a good adsorption process, close to 76.2\% for E2 removal and approximately $55.0 \%$ for EE2. Moreover, the results indicated a probable multi-layered process. Adsorption isotherms were well fitted by Freundlich model. The data were evaluated considering the pseudo-first-order and pseudo-second-order approaches, being the second more significant mechanism in the rate-controlling step. Thermodynamic data revealed that hormones adsorption onto peat is spontaneous under the employed experimental conditions. The results confirmed the potential of this adsorbent to be employed for effluents treatment.
\end{abstract}

Keywords: estradiol; adsorption; peat.

\section{INTRODUÇÃO}

A água é um recurso fundamental para a sobrevivência do homem e para o equilíbrio de todos os ecossistemas de nosso planeta e, nos dias atuais, ela representa uma preocupação mundial diante das ameaças da poluição, do uso insustentável, das mudanças climáticas, das pressões associadas ao uso do solo e do risco de escassez. A presença de micro-organismos patogênicos em águas naturais provoca a morte de mais de 2 milhões de pessoas anualmente em todo o mundo; a maioria crianças com idade abaixo de 5 anos. $\mathrm{O}$ aumento da poluição das águas superficiais e subterrâneas por produtos químicos, com efeitos na vida aquática e na saúde humana, também pode provocar problemas similares ou até mesmo de maior severidade. Mais de um quinto da água doce facilmente acessível é utilizado para fins domésticos, agrícolas e industriais, sendo que a maioria dessas atividades causa a contaminação das águas por uma série de compostos de origem sintética ou geogênica. Nesse contexto, não é surpresa que a preocupação com a poluição das águas por produtos químicos esteja se tornando um dos principais focos de interesse público mundial. ${ }^{1}$

Atualmente, a avaliação dos efeitos dos inúmeros contaminantes naturais e sintéticos que podem estar presentes em águas naturais em concentrações na faixa de $\mathrm{pg} \mathrm{L}^{-1}$ a ng L ${ }^{-1}$ é ainda um grande desafio. ${ }^{2-4}$ Esses compostos representam um risco à saúde dos ecossistemas, considerando sua diversidade e a imensa carência existente no co-

*e-mail: deiaqmc@gmail.com

\#Endereço atual: Depto Química Inorgânica, Instituto de Química, UFRGS, 91501-970 Porto Alegre - RS, Brasil nhecimento detalhado de suas fontes, comportamento, distribuição, níveis tóxicos de concentração, assim como efeitos ambientais. A avaliação de impacto da contaminação química tem estado restrita quase que exclusivamente a produtos convencionais, especialmente agrotóxicos e subprodutos industriais, que mostram persistência no ambiente. Tais produtos, entretanto, representam somente uma parte do potencial poluente. Outros contaminantes, até então negligenciados, vêm chamando a atenção da comunidade científica, sendo denominados contaminantes emergentes. ${ }^{5,6}$

Mesmo presentes em pequenas concentrações os contaminantes emergentes, em algumas situações, podem interferir no sistema endócrino de humanos e de outros animais, afetando a saúde, o crescimento e a reprodução dos mesmos, sendo conhecidos como interferentes endócrinos (IE). Alguns efeitos citados na literatura, tais como diminuição na eclosão de ovos de pássaros, peixes e tartarugas; feminização de peixes machos; indução da síntese de vitoelogenina no plasma de peixes; problemas no sistema reprodutivo de peixes, répteis, pássaros e mamíferos e, alterações no sistema imunológico de mamíferos marinhos, têm sido associados à exposição de diversas espécies aos IE. Em alguns casos, esses efeitos podem conduzir até mesmo ao declínio das populações. Em seres humanos, esses efeitos podem incluir a redução da quantidade de esperma, o aumento da incidência de câncer de mama, de testículo e de próstata, e a endometriose. , $^{3,7}$

Os IE encontram-se principalmente entre as classes de compostos provenientes de produtos ou subprodutos químicos industriais, como aqueles gerados durante a combustão e incineração, ${ }^{8}$ sendo as águas subterrâneas, rios e lagos, os seus principais destinos. ${ }^{9}$ As quatro principais classes de IE (os estrogênios naturais, estrogênios sintéti- 
cos, fitoestrogênios e diversos produtos químicos industriais) são, em geral, designadas com relação ao seu potencial estrogênico, ${ }^{10}$ sendo os hormônios naturais e sintéticos aqueles que apresentam potenciais mais elevados. Entretanto, as quantidades de fitoestrogênios e xenoestrogênios encontradas em águas residuais são frequentemente superiores. ${ }^{11}$

A lista de contaminantes traço ou IE resultantes de atividades antrópicas e encontradas em águas residuais é extensa. ${ }^{12-14}$ Contudo, os hormônios naturais (estrona, $17 \beta$-estradiol e estriol) e sintéticos $(17 \alpha-$ etinilestradiol e mestranol) são os principais causadores de atividades estrogênicas encontrados em corpos aquáticos ${ }^{15}$ e no esgoto bruto ou tratado. Esses IE são excretados através da urina em suas formas biologicamente ativas, ou seja, como conjugados solúveis em água e, em menor proporção, por meio das fezes. Sob condições naturais, esses metabólitos podem ser rapidamente hidrolisados, retornando a sua forma original. ${ }^{16} \mathrm{O}$ hormônio $17 \beta$-estradiol, por exemplo, aporta nos corpos aquáticos devido à frequente utilização como aditivo em alimentos para bovinos, sendo excretado principalmente por meio da urina. Criações industriais de animais, como bovinos e suínos, bem como os efluentes das estações de tratamento de esgotos domésticos, são as principais fontes de $17 \beta$-estradiol para o ambiente. ${ }^{4,17} \mathrm{O}$ hormônio sintético $17 \alpha$-etinilestradiol também é considerado um IE de grande importância, devido a sua ampla utilização em contraceptivos, nas terapias de reposição hormonal e no tratamento do câncer de próstata, sendo assim liberado em grandes quantidades através da urina. De modo geral, os estrogênios sintéticos são muito menos ativos do que os naturais; no entanto, incrementam a estrogenecidade de outros compostos químicos.

A presença de IE em águas superficiais está diretamente relacionada à eficiência dos processos de tratamento de efluentes e esgotos, pelo menos em países onde existe essa prática de saneamento. No Brasil, onde apenas cerca de $50 \%$ dos municípios têm algum tipo de serviço de esgotamento sanitário, ${ }^{18}$ esse cenário torna-se ainda mais preocupante. Dessa forma, invariavelmente, muitos IE são detectados em águas superficiais devido à inadequação dos sistemas de tratamento ou à falta de tratamento de esgotos ou efluentes. ${ }^{16,19}$ Portanto, novas alternativas de descontaminação de efluentes e esgotos que proporcionem a remoção adequada de IE precisam ser desenvolvidas. Os métodos mais utilizados são aqueles que empregam processos de adsorção e processos oxidativos avançados. Apesar dos últimos serem mais eficientes na remoção dos contaminantes, esses podem levar à formação de produtos intermediários, os quais não têm comportamento e efeitos bem conhecidos até o presente momento. ${ }^{20-23}$ Além disso, subprodutos de oxidação indesejáveis, tais como compostos orgânicos halogenados, podem ser formados durante os processos oxidativos que utilizam cloro e ozônio. Por outro lado, a adsorção não resulta em subprodutos de oxidação, mas pode implicar em processos relativamente caros devido à utilização de altas dosagens de carvão ativado, material geralmente empregado em estações de tratamento de efluentes e de esgotos, para remoção de contaminantes orgânicos. ${ }^{24}$

Recentemente, diferentes adsorventes têm sido estudados como uma alternativa tecnológica para a remoção de poluentes emergentes presentes em efluentes aquosos, ${ }^{25-28}$ dentre os quais pode-se destacar a turfa. ${ }^{27,29-33}$ Esse material é relativamente barato e possui boa capacidade de adsorção, devido à presença de grupos funcionais de grande reatividade que atuam nas reações de troca iônica, fazendo com que o potencial de adsorção frente a certas espécies, tais como metais e moléculas orgânicas polares, seja muito alto. ${ }^{34}$ Dentro desse contexto, o presente trabalho teve por objetivo principal avaliar o potencial de adsorção de uma amostra de turfa decomposta com relação aos hormônios $17 \beta$-estradiol e $17 \alpha$-etinilestradiol presentes em solução aquosa. Parâmetros cinéticos, de equilíbrio e termodinâmicos também foram investigados. Além disso, inicialmente aos estudos de adsorção, realizou-se a otimização da técnica de extração em fase sólida (EFS), devido à necessidade de uma etapa de concentração dos analitos após a adsorção em turfa.

\section{PARTE EXPERIMENTAL}

\section{Material e reagentes}

Todos os reagentes utilizados foram de pureza adequada para a realização do trabalho (grau analítico). Os solventes metanol e acetonitrila foram adquiridos da Riedel-de Haen e LabScan, respectivamente. O cloreto de cálcio foi obtido da Fluka. As membranas de filtração de $0,45 \mu \mathrm{m}$ de porosidade foram adquiridas da Millipore. Os padrões dos hormônios 17 $\beta$-estradiol (E2) e 17 $\alpha$-etinilestradiol (EE2) foram adquiridos da Riedel-de Haen e Sigma, respectivamente, apresentando $98 \%$ de pureza. A água utilizada na preparação das amostras e das fases móveis foi obtida a partir de um sistema milli-Q (Millipore).

\section{Extração em fase sólida (EFS)}

A técnica de extração em fase sólida (EFS) empregada na concentração dos hormônios foi baseada nos procedimentos preconizados pela Agência de Proteção Ambiental Norte-americana - U.S. EPA (método 3535A) ${ }^{35}$ Foram utilizados dois cartuchos $(0,5 \mathrm{~g} / 6 \mathrm{~mL})$ contendo diferentes tipos de sorvente: um polímero de estirenodivinilbenzeno com superfície modificada (STRATA-X - Phenomenex) e outro de octadecilsilano (ENVI-18 - Supelco). Todas as EFS foram realizadas utilizando-se um sistema de vácuo tipo manifold da Phenomenex. Anteriormente à aplicação das amostras, os cartuchos foram pré-condicionados com $4 \mathrm{~mL}$ de metanol e posteriormente com

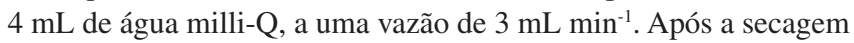
do cartucho, por meio da passagem de ar, um volume de $200 \mathrm{~mL}$ de amostra foi então percolado a $3 \mathrm{~mL} \mathrm{~min}^{-1}$, sob vácuo de $6 \mathrm{kPa}$. Em seguida, os cartuchos foram lavados com $2 \mathrm{~mL}$ de água milli-Q e secos sob vácuo por cerca de 5 min para que pequenas quantidades de água fossem eliminadas. Os hormônios foram eluídos com $4 \mathrm{~mL}$ do solvente adequado, sob vácuo e com uma vazão de $3 \mathrm{~mL} \mathrm{~min}{ }^{-1}$. A escolha do melhor solvente para a eluição dos hormônios na EFS foi feita através de testes de recuperação de analito adicionado $\left(0,1 \mathrm{mg} \mathrm{L}^{-1}\right)$, empregando diferentes solventes ou mistura dos mesmos. Após a eluição, os analitos foram quantificados por cromatografia em fase líquida.

\section{Experimentos de adsorção}

\section{Material adsorvente}

Para a realização dos experimentos de adsorção foi utilizada uma amostra de turfa coletada em uma turfeira em Balneário Arroio do Silva (Santa Catarina, Brasil). ${ }^{27-28,36}$ Essa amostra foi avaliada in situ, empregando-se o modelo proposto por von Post. ${ }^{37}$ Nesse modelo, as turfas são classificadas de acordo com uma escala visual de 10 pontos (de H1 - sem decomposição, até H10 - completamente decomposta), após um teste de campo expedito de espremedura (squeezing). Esse teste consiste em espremer à mão uma amostra fresca e molhada, de modo a observar-se o aspecto do material que flui entre os dedos e daquele que fica retido na mão. A turfa desse trabalho foi classificada como H7 (fortemente decomposta), pois o líquido que fluiu entre os dedos se apresentava muito escuro e os resíduos vegetais que restaram na mão eram praticamente irreconhecíveis. $\mathrm{O}$ percentual médio de decomposição para a maioria das turfas desse tipo (H7) é da ordem de $70 \%$. Turfas com grau de decomposição dessa ordem de grandeza apresentam normalmente um poder de adsorção intermediário, ${ }^{37,38}$ podendo a amostra ser usada como adsorvente frente a soluções aquosas contaminadas por diferentes contaminantes..$^{27-33}$ 


\section{Adsorção}

A adsorção dos hormônios E2 e EE2 foi realizada à temperatura ambiente $\left(\sim 25^{\circ} \mathrm{C}\right)$ e em $\mathrm{pH}$ natural (aproximadamente 5). A força iônica foi mantida constante com $\mathrm{CaCl}_{2} 0,01 \mathrm{~mol} \mathrm{~L}^{-1}$. Para todos os experimentos realizados, um volume de $210 \mathrm{~mL}$ de cada solução contendo o hormônio estudado, com concentração inicial de 0,10 $\mathrm{mg} \mathrm{L}^{-1}$, foi deixado em contato com 50, 75, 100, 150 e $200 \mathrm{mg}$ de turfa, sob agitação magnética constante. A adsorção foi acompanhada por coletas periódicas de $200 \mathrm{~mL}$ de solução por períodos de no máximo 36 h. Após a coleta das soluções, as mesmas foram filtradas e concentradas 50 vezes através do método de EFS.

\section{Determinação dos hormônios}

Os analitos estudados foram detectados e quantificados em um cromatógrafo em fase líquida Jasco 980, equipado com uma coluna de octadecil $(150 \times 4,60 \mathrm{~mm}$ d.i., $5 \mu \mathrm{m}$, Phenomenex $)$ e um detector de absorção na região do UV-Vis. A pressão utilizada foi de 68-70 $\mathrm{kg} \mathrm{cm}^{-2}$ e o comprimento de onda empregado na detecção dos hormônios foi de $280 \mathrm{~nm}$. A fase móvel utilizada foi uma solução de acetonitrila:água $(65: 35 \mathrm{v} / \mathrm{v})$, anteriormente filtrada em membrana $0,45 \mu \mathrm{m}$. As análises foram feitas após a injeção de um volume de amostra de $20 \mu \mathrm{L}$, sob vazão de $0,7 \mathrm{~mL} \mathrm{~min}^{-1}$, sendo o tempo total da corrida cromatográfica de 5 min. A quantificação dos compostos foi realizada através de padronização externa, empregando-se uma curva analítica com sete diferentes valores de concentração: 1,0; 2,0; 3,0; 4,0; 5,0; 7,0 e 9,0 mg L-1, preparadas pela diluição de uma solução estoque de $50 \mathrm{mg} \mathrm{L}^{-1}$. Para cada ponto da curva analítica realizou-se a concentração dos analitos por EFS previamente à análise cromatográfica, de acordo com o procedimento descrito na seção Extração em fase sólida (EFS). A curva analítica foi obtida a partir da média dos valores das áreas dos picos cromatográficos para 3 determinações.

\section{Validação do método}

O desempenho analítico do método foi avaliado por meio dos seguintes parâmetros de mérito: linearidade, precisão, recuperação, limites de detecção (LD) e quantificação (LQ). A determinação desses parâmetros foi realizada de acordo com Ribani e colaboradores ${ }^{39} \mathrm{e}$ as recomendações do INMETRO ${ }^{40} \mathrm{~A}$ linearidade foi determinada com base nos coeficientes de correlação linear $\left(r^{2}\right)$ das curvas analíticas para os hormônios E2 e EE2. A precisão foi estimada pela variância na concentração obtida da análise em 6 réplicas de uma amostra de água superficial fortificada com 2,0 mg de E2 e de EE2, e analisada no mesmo dia pelo mesmo analista. Os testes de recuperação foram feitos em triplicata pela adição de 7,0 mg de cada hormônio em 1,0 L de amostra de água superficial filtrada (membrana 0,45 $\mu \mathrm{m}$ ). O LD foi definido como a menor concentração de E2 e EE2 que pôde ser detectada como um pico cromatográfico com uma razão sinal ruído $(R S R)$ de 3,3. O LQ foi designado como a menor concentração da curva analítica. ${ }^{39,40}$

\section{RESULTADOS E DISCUSSÃO}

\section{Otimização da técnica de extração em fase sólida (EFS)}

Um dos parâmetros mais importantes na aplicação da técnica de EFS é a seleção de um sorvente sólido adequado para a retenção dos analitos, assim como o uso de solventes apropriados para a eluição dos mesmos. ${ }^{41}$ Dependendo do tipo de cartucho utilizado, os percentuais de recuperação dos analitos podem variar de 10 a $90 \% .{ }^{42}$ Atualmente, a EFS aplicada a estrogênios naturais e sintéticos de matrizes ambientais tem sido amplamente realizada por meio do emprego de uma fase estacionária de octadecil ligada ao suporte de sílica fundida (octadecilsilano), sorventes poliméricos e da combinação entre eles. ${ }^{43-}$ ${ }^{45}$ Nesse trabalho, a EFS das amostras aquosas foi realizada com dois tipos diferentes de sorventes: um polímero de estireno-divinilbenzeno com superfície modificada (STRATA-X), e outro de octadecilsilano (ENVI-18). A Tabela 1 apresenta os valores de recuperação obtidos nos experimentos de EFS, para ambos os hormônios estudados, empregando diferentes tipos de eluentes.

Tabela 1. Resultados de recuperação obtidos nos experimentos de EFS para os hormônios E2 e EE2

\begin{tabular}{|c|c|}
\hline Eluente & Recuperação (\%) \\
\hline \multicolumn{2}{|l|}{ E2 } \\
\hline \multicolumn{2}{|l|}{ STRATA-X } \\
\hline Acetonitrila & $14,8 \pm 0,6$ \\
\hline Hexano & $26,7 \pm 1,5$ \\
\hline Metanol & $56,3 \pm 1,5$ \\
\hline Tetra-hidrofurano & $110 \pm 3,4$ \\
\hline Tetra-hidrofurano:metanol $(50: 50 \mathrm{v} / \mathrm{v})$ & $75,8 \pm 4,8$ \\
\hline \multicolumn{2}{|l|}{ ENVI-18 } \\
\hline Metanol & $81,6 \pm 1,2$ \\
\hline Acetonitrila & $93,5 \pm 2,0$ \\
\hline \multicolumn{2}{|l|}{ EE2 } \\
\hline \multicolumn{2}{|l|}{ ENVI-18 } \\
\hline Acetonitrila & $71,9 \pm 1,9$ \\
\hline Tetra-hidrofurano:acetonitrila $(20: 80 \mathrm{v} / \mathrm{v})$ & $76,9 \pm 2,8$ \\
\hline Tetra-hidrofurano:acetonitrila $(50: 50 \mathrm{v} / \mathrm{v})$ & $77,0 \pm 3,0$ \\
\hline Acetonitrila:água $(65: 35 \mathrm{v} / \mathrm{v})$ & $71,8 \pm 0,9$ \\
\hline
\end{tabular}

Para o hormônio E2 testou-se inicialmente o cartucho STRATAX. Os resultados de recuperação para esse cartucho ficaram entre 14,8 e $110 \%$. Para os testes empregando os eluentes acetonitrila, hexano e metanol, os percentuais de recuperação encontrados $(14,8$; 26,7 e $56,3 \%$, respectivamente) ficaram abaixo da faixa aceitável para métodos cromatográficos, que está situada entre 70 e $120 \%{ }^{39}$ Devido à forte interação do hormônio E2 com a fase estacionária de estireno-divinilbenzeno modificada presente no cartucho STRATA-X, o solvente apropriado para a remoção do analito deve ter uma polaridade adequada para a eficiência do processo. Essa relação pôde ser observada pela tendência dos dados de recuperação para o hormônio $\mathrm{E} 2$, onde os solventes mais apolares (acetonitrila e hexano) apresentaram os menores valores de recuperação. Apesar dos resultados para os eluentes tetra-hidrofurano $(110 \%)$ e tetra-hidrofurano:metanol $(50: 50 \mathrm{v} / \mathrm{v})(75,8 \%)$ ficarem dentro da faixa de recuperação aceitável, esses não foram considerados satisfatórios para as análises, pois a utilização do tetra-hidrofurano como eluente gerou interferências durante a análise cromatográfica. Diante desses aspectos, o cartucho ENVI-18 também foi avaliado para ambos os hormônios.

Como pode ser observado na Tabela 1 , os resultados obtidos para o hormônio E2 empregando o cartucho ENVI-18, com os solventes metanol e acetonitrila, ficaram dentro da faixa de recuperação aceitável em todos os testes. ${ }^{39} \mathrm{O}$ melhor resultado de recuperação foi obtido com o eluente acetonitrila $(93,5 \%)$, o qual foi empregado nos experimentos de adsorção desse hormônio pela amostra de turfa. Comparando-se os dados para o mesmo tipo de fase estacionária, é possível constatar-se que a faixa de valores de recuperação (de 71,8 a 77,0\%) para o hormônio EE2 ficou menor do que para o hormônio E2, mas, mesmo assim, esses resultados foram considerados satisfatórios. ${ }^{39}$ Essa diferença pode ser explicada pela maior lipofilicidade do hormônio E2 com relação ao hormônio EE2, acarretando em uma maior afinidade com a fase móvel. Resultados semelhantes também foram obtidos por Labadie e Budzinski. ${ }^{43}$ Com relação ao hormônio EE2, os melhores resultados de recuperação foram obtidos com o eluente tetra-hidrofurano:acetonitrila 
Tabela 2. Resultados relativos aos parâmetros de mérito obtidos após a otimização da técnica de EFS, para os hormônios E2 e EE2

\begin{tabular}{lcccccc}
\hline Composto & Tempo de retenção $(\mathrm{min})$ & Linearidade $\left(\mathrm{r}^{2}\right)$ & DPR $(\%)$ & LD $\left(\mathrm{mg} \mathrm{L}^{-1}\right)$ & LQ $\left(\mathrm{mg} \mathrm{L}^{-1}\right)$ & Recuperação $(\%)$ \\
\hline E2 & 3,58 & 0,9995 & 2,41 & 0,58 & 1,00 & $95,3 \pm 2,0$ \\
EE2 & 4,25 & 0,9983 & 6,64 & 0,72 & 1,00 & $86,7 \pm 3,0$ \\
\hline
\end{tabular}

$\mathrm{DPR}=$ Desvio padrão relativo; $\mathrm{LD}=$ Limite de detecção; $\mathrm{LQ}=$ Limite de quantificação.

$(20: 80$ e $50: 50 \mathrm{v} / \mathrm{v})(76,9 \mathrm{e} 77,0 \%$, respectivamente). No entanto, optouse por trabalhar com o eluente acetonitrila:água $(65: 35 \mathrm{v} / \mathrm{v})$, o qual apresentou resultado de recuperação satisfatório $(71,8 \%)$ e semelhante ao eluente acetonitrila (71,9\%), além de ter a mesma composição que a fase móvel empregada na análise cromatográfica.

\section{Parâmetros de mérito}

Na Tabela 2 são apresentados os resultados relativos aos parâmetros de mérito para o método avaliado. A determinação da área relativa de cada nível da curva analítica, para ambos os compostos, foi feita em triplicata e a média dos desvios padrão relativos foi inferior a $3 \%$. As duas curvas analíticas apresentaram boa linearidade dentro da faixa de concentração estudada (1-9 mg L $\left.\mathrm{L}^{-1}\right)$, com valores de $r^{2}$ de 0,9995 para o hormônio E2, e de 0,9983 para o hormônio EE2, demonstrando a excelente correlação entre as áreas dos picos e a concentração dos padrões. Os tempos de retenção para os hormônios E2 e EE2 foram de 3,58 e 4,25 min, respectivamente. Uma boa precisão foi igualmente obtida, como pode ser comprovado pelos baixos valores de desvio padrão relativo (DPR): de 2,41\% para o hormônio E2 e 6,64\% para o hormônio EE2.

Os valores de limite de detecção (LD) e de quantificação (LQ) para cada composto estudado foram calculados pelo método da relação sinal-ruído e pelo menor nível de concentração de padrão na curva analítica, respectivamente. ${ }^{39,40}$ Como pode ser observado na Tabela 2, os valores de LD foram de $0,58 \mathrm{mg} \mathrm{L}^{-1}$ para o hormônio E2, e de $0,72 \mathrm{mg} \mathrm{L}^{-1}$ para o hormônio EE2, o que demonstra a boa sensibilidade do método de análise empregado.

$\mathrm{Na}$ ausência de material de referência certificado, a exatidão do método foi avaliada pela adição de uma quantidade conhecida de padrão em amostras de água superficial, sendo feita uma correlação direta do valor determinado após o teste, com o valor de padrão adicionado (7,0 mg). Como pode ser observado na Tabela 2 , os dados de recuperação do padrão adicionado foram de $95,3 \pm 2,0 \%$ para o hormônio E2 e de 86,7 \pm 3,0\% para o hormônio EE2. Por estarem dentro da faixa de recuperação aceitável para a determinação de compostos orgânicos traço (de 70 a 120\%), os valores de recuperação aqui encontrados foram considerados satisfatórios. ${ }^{39}$

\section{Adsorção dos hormônios 17 $\beta$-estradiol e 17 $\alpha$-etinilestradiol}

\section{Efeito do tempo de contato e massa de turfa na adsorção}

A adsorção dos hormônios E2 e EE2, a partir de soluções aquosas pela amostra de turfa, foi realizada com cinco massas diferentes de adsorvente (50, 75, 100, 150 e $200 \mathrm{mg}$ ), usando diferentes tempos de adsorção e com concentração inicial de hormônio de $0,10 \mathrm{mg} \mathrm{L}^{-1}$. Em geral, o processo de adsorção foi mais intenso no início do experimento devido à elevada afinidade entre a turfa e os hormônios nas duas primeiras horas (Figuras 1a e 1b), sendo o valor máximo de remoção encontrado somente depois de algumas horas de tempo de contato. Após a adsorção, a quantidade de hormônio adsorvida, assim como a concentração de hormônio em solução (Tabela 3), permaneceu praticamente constante para todos os casos, indicando que o limite de remoção já foi atingido no experimento de menor massa de turfa, sendo o fator limitante o tempo de exposição e as energias de interação entre a turfa, os hormônios e o solvente. Quando a solução se torna
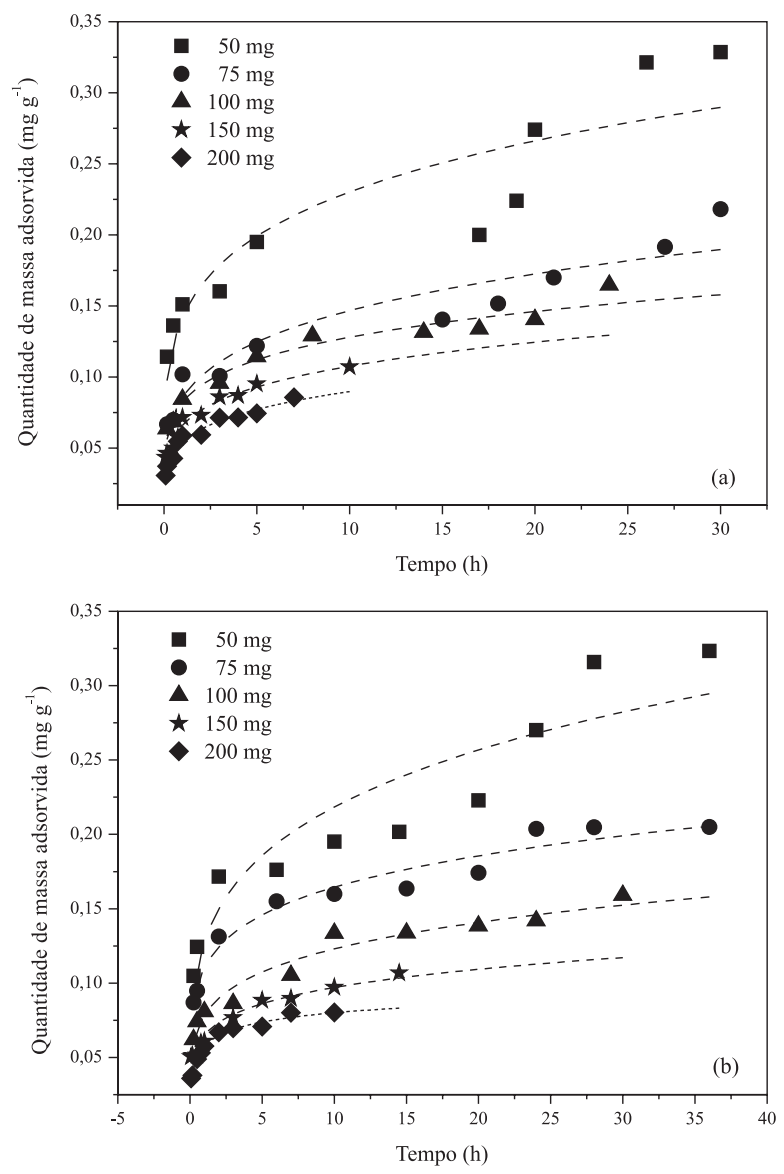

Figura 1. Quantidade de massa adsorvida de (a) E2 e (b) EE2 pelas diferentes massas de turfa

diluída, a estabilização da concentração de soluto tende a aumentar e como consequência desse resultado há uma maior dificuldade em se remover os últimos traços do soluto da solução. ${ }^{46}$

Outro fator importante a ser mencionado diz respeito ao perfil dos gráficos de quantidade de hormônio adsorvida em função do tempo (Figuras 1a e 1b). Para todas as massas de turfa estudadas, é possível observar uma provável adsorção da primeira camada adsorvedora, para ambos os hormônios, nas primeiras horas do processo. Em seguida, outro processo de adsorção parece estar acontecendo, resultando em um segundo platô em maiores tempo de contato. Esse platô não é evidente no caso dos experimentos envolvendo quantidades maiores de turfa $(100,150$ e 200 mg) devido à maior disponibilidade de sítios ativos. Essas são características típicas de processos onde ocorre a saturação de uma camada e a subsequente formação de outras. Sendo assim, grande parte do hormônio em solução é rapidamente transferida para a camada superficial da turfa nas primeiras horas do processo. Um novo processo de adsorção, com a saturação da segunda camada adsorvedora parece ocorrer em tempos de contato mais longos, especialmente para os experimentos de 50 e $75 \mathrm{mg}$ de turfa. Resultados similares foram encontrados por Fernandes e colaboradores ${ }^{28}$ empregando o mesmo adsorvente, na adsorção do corante azul de metileno. Nesse estudo, o corante foi 
Tabela 3. Resultados relativos à remoção dos hormônios E2 e EE2 pela amostra de turfa

\begin{tabular}{|c|c|c|c|c|c|}
\hline \multirow[t]{2}{*}{ Parâmetros de adsorção } & \multicolumn{5}{|c|}{ Massa de turfa (mg) } \\
\hline & 50 & 75 & 100 & 150 & 200 \\
\hline & & & E2 & & \\
\hline Tempo (h) & 30 & 30 & 24 & 10 & 7 \\
\hline Porcentagem de remoção (\%) & 73,1 & 72,7 & 73,2 & 71,5 & 76,2 \\
\hline Quantidade de massa adsorvida (mg) & 0,016 & 0,016 & 0,016 & 0,016 & 0,017 \\
\hline Quantidade de massa adsorvida por grama de turfa $\left(\mathrm{mg} \mathrm{g}^{-1}\right)$ & 0,200 & 0,141 & 0,132 & 0,107 & 0,086 \\
\hline \multirow[t]{2}{*}{ Concentração após adsorção $\left(\mathrm{mg} \mathrm{L}^{-1}\right)$} & 0,022 & 0,022 & 0,022 & 0,023 & 0,018 \\
\hline & & & EE2 & & \\
\hline Tempo (h) & 36 & 36 & 30 & 14 & 10 \\
\hline Porcentagem de remoção (\%) & 55,0 & 52,3 & 54,1 & 54,5 & 54,5 \\
\hline Quantidade de massa adsorvida (mg) & 0,016 & 0,015 & 0,016 & 0,016 & 0,016 \\
\hline Quantidade de massa adsorvida por grama de turfa $\left(\mathrm{mg} \mathrm{g}^{-1}\right)$ & 0,218 & 0,173 & 0,142 & 0,105 & 0,083 \\
\hline Concentração após adsorção $\left(\mathrm{mg} \mathrm{L}^{-1}\right)$ & 0,023 & 0,027 & 0,024 & 0,024 & 0,024 \\
\hline
\end{tabular}

adsorvido pela superfície externa da turfa, em um processo bastante rápido. Quando a adsorção da camada externa da turfa atingiu a saturação, as moléculas do corante moveram-se através dos poros para o interior das partículas e foram adsorvidas pela superfície interna da turfa. Considerando todos esses aspectos, a adsorção de ambos os hormônios aqui estudada provavelmente ocorreu em múltiplas camadas.

Os valores encontrados para a quantidade de hormônio adsorvida no equilíbrio por grama de turfa $\left(q_{e}\right)$ foram de $0,086-0,200 \mathrm{mg} \mathrm{g}^{-1}$ para o E2 e de 0,083-0,218 $\mathrm{mg} \mathrm{g}^{-1}$ para o EE2 (Tabela 3). Nesse caso foi considerada, para efeito de comparação, a formação de uma primeira camada. $\mathrm{O}$ aumento de massa de turfa proporcionou uma diminuição na quantidade de massa adsorvida para ambos os hormônios. Os dados de $q_{e}$ encontrados são considerados significativos quando comparados a outros estudos de adsorção de E2 e EE2, empregando diferentes adsorventes. Chen e colaboradores ${ }^{47}$ por exemplo, avaliaram o poder de remoção do E2 de soluções aquosas utilizando resíduo de carvão e obtiveram valores máximos de $q_{e}$ de $0,0498 \mathrm{mg} \mathrm{g}^{-1}$. A adsorção de EE2 pelo fulereno ${ }^{48}$ apresentou um valor de $q_{e}$ de $0,139 \mathrm{mg} \mathrm{g}^{-1}$. Todos esses aspectos demonstram a alta capacidade de adsorção da turfa aqui estudada.

As Figuras 2 e 3 mostram, respectivamente, a variação da porcentagem de remoção dos hormônios E2 e EE2 em função do tempo, para diferentes massas de turfa. Em todos os experimentos, a concentração inicial de hormônio foi mantida constante. Esse valor foi escolhido para que, após a adsorção, os compostos pudessem ainda ser detectados pela análise cromatográfica. Para todos os casos, observa-se um aumento no percentual de remoção do hormônio com o aumento do tempo de contato. Além disso, um aumento mais significativo na remoção é observado nas primeiras horas, em todos os experimentos. Com o passar do tempo e para massas maiores de turfa, ocorre uma turvação na solução devido à dissolução da matéria orgânica. Essa solução absorve na mesma faixa de comprimento de onda que os hormônios estudados. Para avaliar essa questão, foi realizado um controle (branco) para todas as massas de turfa estudadas, contendo somente o material adsorvente e água milli-Q. Dessa forma, os experimentos foram mantidos até determinados tempos de contato, de tal forma que a quantidade de matéria orgânica dissolvida em solução não interferisse na quantificação dos hormônios.

Para todas as massas de turfa utilizadas, o percentual de remoção chegou a valores máximos de adsorção de 76,2 e de 55,0\%, respectivamente, para os hormônios E2 e EE2 (Tabela 3), já descontando os valores de recuperação para cada respectivo composto obtido por EFS. Para ambos os hormônios, o aumento da massa de turfa promoveu um ligeiro aumento na porcentagem de remoção e uma
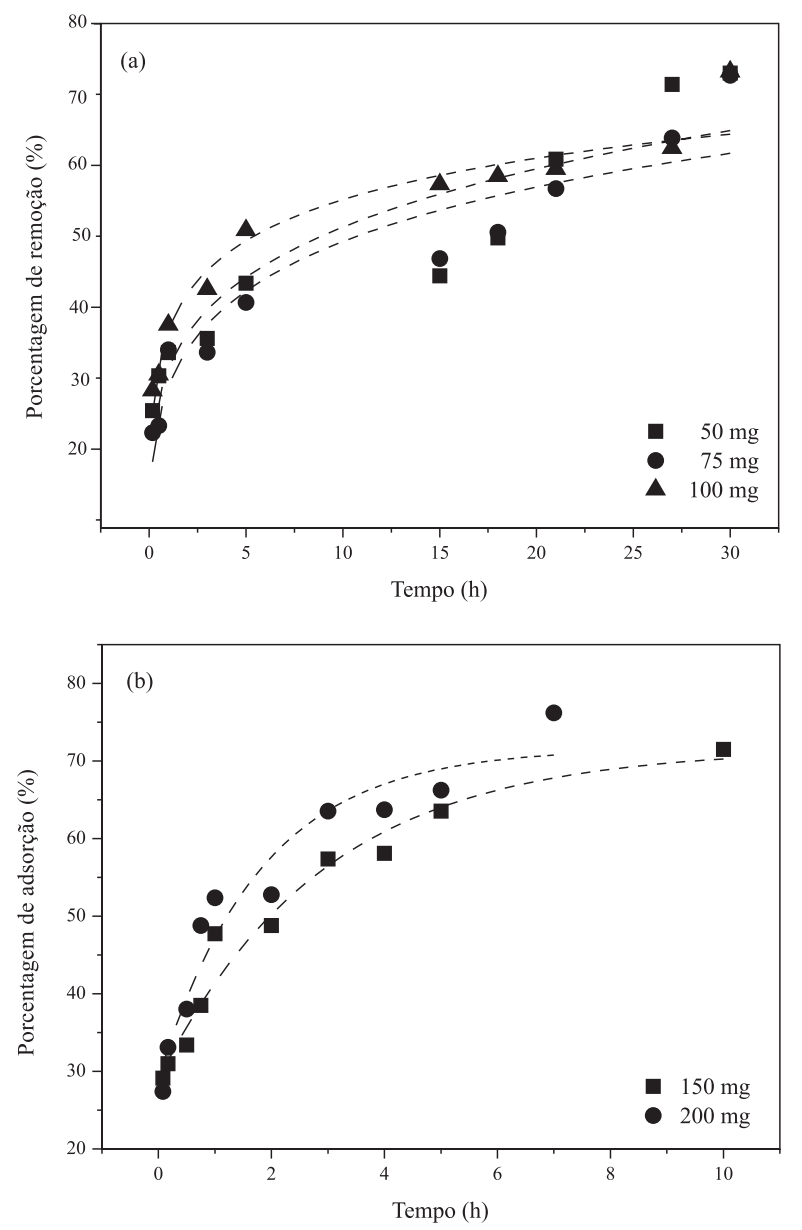

Figura 2. Porcentagem de remoção do hormônio E2 pela amostra de turfa de: (a) 50, 75 e $100 \mathrm{mg} \mathrm{e}$ (b) 150 e $200 \mathrm{mg}$

diminuição significativa dos tempos de adsorção (Figuras 2 e 3). Essas características estão associadas à presença de uma maior quantidade de adsorvente em contato com a solução de hormônios, ocasionando, dessa forma, uma remoção mais rápida dos mesmos pela turfa. Em todos os experimentos, a porcentagem de adsorção foi menor para o hormônio EE2 do que para o hormônio E2. Essa diferença pode estar associada às particularidades estruturais de cada hormônio. $\mathrm{O}$ hormônio EE2 possui características mais apolares do que o hormônio E2, devido à presença do grupo etinil em sua estrutura, podendo dessa forma ter menor afinidade pela amostra de turfa. 

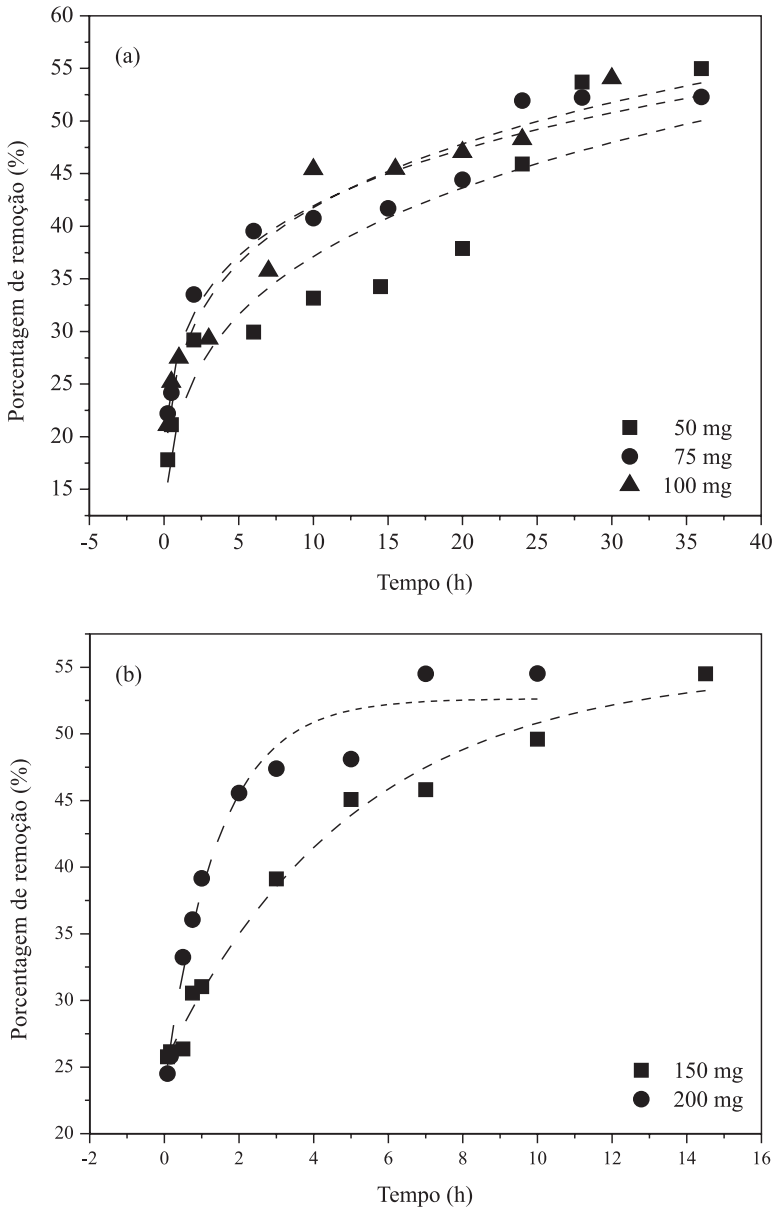

Figura 3. Porcentagem de remoção do hormônio EE2 pela amostra de turfa de: (a) 50, 75 e $100 \mathrm{mg}$ e (b) 150 e $200 \mathrm{mg}$

Diferentes tipos de adsorventes têm sido avaliados para a remoção dos hormônios E2 e EE2 presentes em soluções aquosas. ${ }^{25,33,49,50}$ Em outro experimento, ${ }^{49}$ usando carvão ativado como material adsorvente para remover os hormônios E2, EE2 e bisfenol A, a seguinte tendência de remoção (levando-se em consideração o coeficiente de partição octanol/água $\left.-K_{o w}\right)$ foi observada: E2 $(3,1-4,0)>$ EE2 $(3,7-3,9)>$ bisfenol A $(3,3)\left(\log K_{o w}\right.$ entre parênteses). Segundo esses autores, ${ }^{49}$ a maior porcentagem de remoção do hormônio E2 (quando comparada aos hormônios EE2 e bisfenol A) pode ser atribuída ao seu maior valor de $K_{o w}$. Nos últimos anos, o $K_{o w}$ vem sendo muito utilizado em várias áreas, com um número elevado de publicações divulgando a sua correlação com outras propriedades físicas, químicas e biológicas dos compostos, além do sucesso de seu uso na estimativa de fatores de bioconcentração, coeficiente de partição carbono orgânico/água, toxicidade e solubilidade. ${ }^{51}$ Diante desses aspectos, a maior solubilidade do hormônio E2, como consequência de seu maior valor de $K_{o w}$, também contribuiu para a maior porcentagem de remoção desse composto pela amostra de turfa aqui estudada.

\section{Isotermas de equilíbrio de adsorção}

Em um processo de adsorção, as isotermas são importantes para descrever como as moléculas de adsorvato estão distribuídas entre as fases líquida e sólida no equilíbrio. $\mathrm{O}$ equilíbrio de um dado sistema é alcançado quando não há mudanças nas concentrações do adsorvato em ambas as fases desse sistema. $\mathrm{O}$ equilíbrio reflete a capacidade e/ou a afinidade de um adsorvente por um adsorvato, sob um dado conjunto de condições a que o sistema é submetido. ${ }^{52}$
Assim, a correlação dos dados de equilíbrio por meio de equações teóricas ou empíricas é essencial para o delineamento de sistemas de adsorção.$^{53}$ Nesse estudo, as isotermas de Langmuir ${ }^{54}$ e Freundlich ${ }^{55}$ foram utilizadas para avaliar o processo de adsorção.

O modelo de Langmuir assume que a adsorção é homogênea e ocorre em sítios específicos do adsorvente formando uma monocamada. Além disso, as moléculas adsorvidas em um sítio de adsorção não devem afetar a adsorção das demais moléculas. A forma linear da isoterma de Langmuir é dada pela Equação 1:

$$
\frac{C_{e}}{q_{e}}=\frac{1}{Q K_{L}}+\frac{C_{e}}{Q}
$$

onde $C_{e}$ é a concentração de equilíbrio do adsorvato $\left(\mathrm{mg} \mathrm{L}^{-1}\right) ; q_{e}$ é a quantidade de adsorvato adsorvida no equilíbrio $\left(\mathrm{mg} \mathrm{g}^{-1}\right)$; e $K_{L}\left(\mathrm{~L} \mathrm{mg}^{-1}\right)$ e $Q\left(\mathrm{mg} \mathrm{g}^{-1}\right)$ são parâmetros de Langmuir que estão relacionados à afinidade dos sítios de adsorção e à quantidade máxima de adsorvato necessária à formação da monocamada, respectivamente.

O modelo de Freundlich, por outro lado, considera que a adsorção ocorre em superfícies heterogêneas, não estando limitado à formação de uma monocamada. ${ }^{56} \mathrm{~A}$ forma linear da isoterma de Freundlich é dada pela Equação 2:

$$
\ln q_{e}=\ln K_{F}+\frac{1}{n} \ln C_{e}
$$

onde $K_{F}\left(\mathrm{~L} \mathrm{mg}^{-1}\right)$ é a constante de Freundlich que está relacionada à capacidade de adsorção, e $n$ é um parâmetro que diz respeito à intensidade de adsorção. Além disso, esses dois parâmetros podem indicar se a natureza do processo de adsorção é favorável ou desfavorável. ${ }^{57}$

Os resultados da adsorção dos hormônios E2 e EE2 pela turfa não foram descritos adequadamente pelo modelo de Langmuir, uma vez que a adsorção não se limitou a apenas uma monocamada. Os gráficos de $C / q_{e}$ versus $C_{e}$ não foram lineares e, consequentemente, os valores de $r^{2}$ não foram satisfatórios. Por outro lado, o modelo de Freundlich ajustou bem os resultados de adsorção para ambos os hormônios, com valores de $r^{2}$ de 0,9280 para E2 e de 0,9944 para EE2. Os valores de $K_{F}$ e $n$, calculados por meio da Equação 2 e pelas isotermas de Freundlich (Figura 4), estão apresentados na Tabela 4. Os valores de $K_{F}$ encontrados para a amostra de turfa nos estudos com os hormônios E2 e EE2 foram de 1,10 e 4,34 $\mathrm{L} \mathrm{mg}^{-1}$, respectivamente. Esses valores, quando comparados àqueles publicados por Song e colaboradores,${ }^{58}$ indicam a alta capacidade de

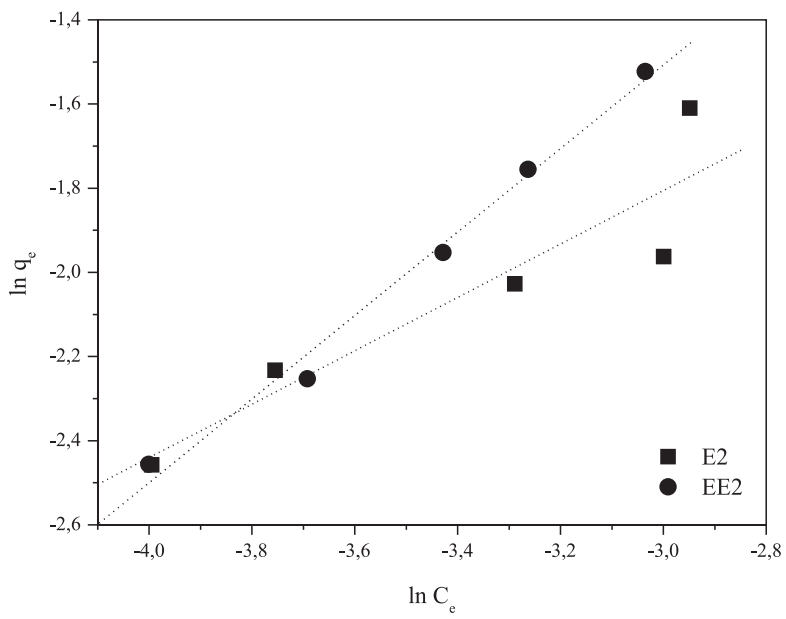

Figura 4. Isotermas de Freundlich para os hormônios E2 e EE2 
Tabela 4. Parâmetros de Freundlich para a adsorção dos hormônios E2 e EE2 pela amostra de turfa

\begin{tabular}{cccccc}
\hline & E2 & \multicolumn{4}{c}{ EE2 } \\
$K_{F}\left(\mathrm{~L} \mathrm{mg}^{-1}\right)$ & $n$ & $r^{2}$ & $K_{F}\left(\mathrm{~L} \mathrm{mg}^{-1}\right)$ & $n$ & $r^{2}$ \\
\hline 1,10 & 1,57 & 0,9280 & 4,34 & 1,01 & 0,9944 \\
\hline
\end{tabular}

adsorção da turfa com relação aos dois compostos estudados. Nos sistemas avaliados, os valores de $n$ foram de 1,57 para o hormônio E2 e de 1,01 para o hormônio EE2, indicando que a intensidade de adsorção é favorável para todas as massas de turfa estudadas. Todos esses aspectos, mais uma vez, sugerem que o processo de adsorção dos hormônios pela turfa estudada ocorre em múltiplas camadas.

\section{Parâmetros cinéticos}

A cinética de adsorção dos hormônios E2 e EE2 pela amostra de turfa foi avaliada por meio das equações de pseudoprimeira ordem $^{59}$ (Equação 3) e pseudossegunda ordem ${ }^{60}$ (Equação 4) abaixo representadas:

$$
\begin{gathered}
\ln \left(q_{e}-q_{t}\right)=\ln q_{e}-k_{1} t \\
\frac{t}{q_{t}}=\frac{1}{k_{2} q_{e}^{2}}+\frac{1}{q_{e}} t
\end{gathered}
$$

onde $q_{t}\left(\mathrm{mg} \mathrm{g}^{-1}\right)$ é a quantidade de hormônio adsorvida no tempo $\mathrm{t}$ (h) e $k_{l}\left(\mathrm{~h}^{-1}\right)$ e $k_{2}\left(\mathrm{~g} \mathrm{mg}^{-1} \mathrm{~h}^{-1}\right)$ são as constantes de velocidade de adsorção de pseudoprimeira ordem e pseudossegunda ordem, respectivamente.

Os parâmetros apresentados na Tabela 5 sugerem um processo de adsorção de pseudossegunda ordem para ambos os sistemas estudados. Os valores de $r_{s}^{2}$ foram maiores do que 0,9940 para todas as massas de turfa estudadas. Uma adsorção de pseudossegunda ordem indica que o processo é dependente tanto da quantidade de hormônio, quanto dos sítios ativos presentes na turfa. Resultados similares também foram observados na adsorção do azul de metileno por uma amostra de turfa ${ }^{28}$ e na adsorção do corante vermelho astrazon por resíduo mineral de mineração de carvão. ${ }^{61}$ Os valores de $k_{2}$ ficaram na faixa de 9,64-43,7 $\mathrm{g} \mathrm{mg}^{-1} \mathrm{~h}^{-1}$ para o E2 e de 8,05-29,8 $\mathrm{g} \mathrm{mg}^{-1} \mathrm{~h}^{-1}$ para o $\mathrm{EE} 2$, sendo o maior valor encontrado para o experimento empregando $200 \mathrm{mg}$ de turfa para adsorver o hormônio E2.

Tabela 5. Parâmetros cinéticos da remoção dos hormônios E2 e EE2 pela amostra de turfa

\begin{tabular}{lcccc}
\hline $\begin{array}{l}\text { Massa de } \\
\text { turfa }(\mathrm{mg})\end{array}$ & $\begin{array}{c}k_{2} \\
\left(\mathrm{~g} \mathrm{mg}^{-1} \mathrm{~h}^{-1}\right)\end{array}$ & $r_{s}^{2}$ & $\begin{array}{c}k_{2} \\
\left(\mathrm{~g} \mathrm{mg}^{-1} \mathrm{~h}^{-1}\right)\end{array}$ & $r_{s}^{2}$ \\
\hline 50 & 9,64 & 0,9994 & 8,05 & 0,9963 \\
75 & 10,9 & 0,9975 & 13,3 & 0,9988 \\
100 & 11,3 & 0,9975 & 15,6 & 0,9994 \\
150 & 28,0 & 0,9941 & 17,7 & 0,9952 \\
200 & 43,7 & 0,9968 & 29,8 & 0,9994 \\
\hline
\end{tabular}

\section{Parâmetros termodinâmicos}

Os valores associados à energia livre de Gibbs foram calculados de acordo com as Equações 5 e 6 :

$$
\begin{gathered}
K_{d}=\frac{C_{a d, e}}{C_{e}} \\
\Delta G=-R T \ln K_{d}
\end{gathered}
$$

onde $C_{a d, e}\left(\mathrm{mg} \mathrm{L}^{-1}\right)$ é concentração dos hormônios na turfa no equilíbrio; $K_{d}$ é a constante de equilíbrio aparente; $\Delta G\left(\mathrm{~J} \mathrm{~mol}^{-1}\right)$ é a energia livre de Gibbs; $R\left(\mathrm{~J} \mathrm{~K}^{-1} \mathrm{~mol}^{-1}\right)$ é a constante universal dos gases e $T$ (K) é a temperatura absoluta do sistema.

Um valor negativo para $\Delta G$ indica uma natureza espontânea do processo de adsorção, não necessitando de valores de energia de ativação elevados para a adsorção ocorrer. ${ }^{61,62}$ Os resultados de $\Delta G$ apresentados na Tabela 6 diminuem à medida que a massa de adsorvente aumenta, indicando uma maior espontaneidade da adsorção devido a maiores possibilidades para que as moléculas dos hormônios sejam acomodadas nos sítios ativos da turfa. Todas essas questões estão relacionadas diretamente com a entropia $(\Delta S)$ do processo. Portanto, a contribuição de $\Delta S$ na Equação 7 aumenta consideravelmente:

$$
\Delta G^{\circ}{ }_{a d s}=\Delta H_{a d s}^{\circ}-T \Delta S_{a d s}^{\circ}
$$

Se a contribuição da entalpia $(\Delta H)$ for desprezada, a Equação 7 pode ser reduzida à Equação 8 e, consequentemente, os valores de $\Delta S$ podem ser estimados:

$$
-\frac{\Delta G_{a d s}^{\circ}}{T}=\Delta S^{\circ}{ }_{a d s}
$$

Os valores de $\Delta S$ apresentados na Tabela 6 mostram que há um aumento na entropia do processo de adsorção com o aumento da quantidade de turfa usada. Um valor positivo e crescente para entropia corrobora o aumento no número de sítios ativos disponíveis com o aumento da massa de turfa empregada. Esse aumento no número de sítios ativos implica em uma maior aleatoriedade, ou seja, em um número maior de opções para que as moléculas de hormônio possam ser adsorvidas.

\section{CONCLUSÕES}

A técnica de análise empregada foi adequada para a determinação dos hormônios E2 e EE2, resultando em valores de $r^{2}$ de 0,9995 e de 0,9983, respectivamente, o que demonstra a boa linearidade na faixa de concentração estudada. Essas informações podem ser comprovadas

\begin{tabular}{|c|c|c|c|c|c|c|}
\hline \multirow[b]{2}{*}{ Massa de turfa (mg) } & \multicolumn{3}{|c|}{ E2 } & \multicolumn{3}{|c|}{ EE2 } \\
\hline & $\begin{array}{c}K_{d} \\
\left(\mathrm{~L} \mathrm{~g}^{-1}\right)\end{array}$ & $\begin{array}{c}\Delta G \\
\left(\mathrm{~kJ} \mathrm{~mol}^{-1}\right)\end{array}$ & $\begin{array}{c}\Delta S \\
\left(\mathrm{~J} \mathrm{~K}^{-1} \mathrm{~mol}^{-1}\right)\end{array}$ & $\begin{array}{c}K_{d} \\
\left(\mathrm{~L} \mathrm{~g}^{-1}\right)\end{array}$ & $\begin{array}{c}\Delta G \\
\left(\mathrm{~kJ} \mathrm{~mol}^{-1}\right)\end{array}$ & $\begin{array}{c}\Delta S \\
\left(\mathrm{~J} \mathrm{~K}^{-1} \mathrm{~mol}^{-1}\right)\end{array}$ \\
\hline 50 & 3,82 & 0,24 & $-0,799$ & 4,54 & $-0,19$ & 0,648 \\
\hline 75 & 2,82 & $-0,02$ & 0,066 & 4,52 & $-1,18$ & 3,976 \\
\hline 100 & 3,53 & $-1,29$ & 4,318 & 4,38 & $-1,82$ & 6,105 \\
\hline 150 & 4,58 & $-2,94$ & 9,859 & 4,22 & $-2,73$ & 9,176 \\
\hline 200 & 4,66 & $-3,69$ & 12,38 & 4,06 & $-3,35$ & 11,25 \\
\hline
\end{tabular}
pelos baixos valores de DPR (E2 $=2,41 \%$ e EE2 $=6,64 \%)$. Os valores de LD e LQ ficaram na faixa de $\mathrm{mg} \mathrm{L}^{-1}$ e os resultados de recuperação foram de 95,3 $\pm 2,0 \%$ para o hormônio E2 e de 86,7 $\pm 3,0 \%$ para o hormônio EE2, sendo considerados satisfatórios.

Tabela 6. Parâmetros termodinâmicos da remoção dos hormônios E2 e EE2 pela amostra de turfa 
A amostra de turfa empregada mostrou-se eficiente na adsorção dos hormônios, sendo os percentuais de remoção de até 76,2\% para o hormônio E2, e de 55,0\% para o hormônio EE2. Para as soluções contendo maiores massas de turfa, o tempo de contato para atingir os mesmos valores de porcentagem de remoção foi menor do que para as soluções contendo menores massas. O modelo de Freundlich ajustou melhor os dados de adsorção dos hormônios E2 e EE2, demonstrando que esse processo ocorreu em múltiplas camadas. Os resultados também foram avaliados pelos modelos cinéticos de pseudoprimeira ordem e pseudossegunda ordem, sendo o último aquele que melhor representou a cinética do processo de adsorção dos hormônios pela turfa. Os valores dos parâmetros termodinâmicos revelaram que a adsorção dos hormônios pela turfa é espontânea nas condições experimentais empregadas. De maneira geral, os resultados obtidos mostram que o grau de adsorção da turfa abre perspectivas para a sua utilização na remoção desses contaminantes em efluentes ou ainda em águas residuais.

\section{AGRADECIMENTOS}

Ao suporte financeiro oferecido pelo Conselho Nacional de Desenvolvimento Científico e Tecnológico (CNPq, Brasil).

\section{REFERÊNCIAS}

1. Schwarzenbach, R. P.; Escher, B. I.; Fenner, K.; Hofstetter, T. B.; Johnson, C. A.; von Gunten, U.; Wehrli, B.; Science 2006, 313, 1072.

2. Ohe, T.; Watanabe, T.; Wakabayashi, K.; Mutat. Res., Rev. Mutat. Res. 2004, 567, 109.

3. Bila, D. M.; Dezotti, M.; Quim. Nova 2007, 30, 651.

4. Ghiselli, G.; Jardim, W. F.; Quim. Nova 2007, 30, 695.

5. Daughton, C. G.; Ternes, T. A.; Environ. Health Perspectives 1999, 107, 907.

6. U.S. EPA - United States Environmental Protection Agency; Region/ ORD Workshop on Emerging Pollutants, Summary Report, 2003.

7. Larsson, D. G. J.; Adolfsson-Erici, M.; Parkkone, J.; Petersson, M.; Berg, A. H.; Olsson, P. E.; Förlin, L.; Aquat. Toxicol. 1999, 45, 91.

8. Ying, G. G.; Williams, B.; Kookana, R.; Environ. Int. 2002, 28, 215.

9. Esperanza, M.; Suidan, M. T.; Nishimura, F.; Wang, Z. M.; Sorial, G. A.; Zaffiro, A.; McCauley, P.; Brenner, R.; Sayles, G.; Environ. Sci. Technol. 2004, 38, 3028 .

10. Servos, M. R.; Water Qual. Res. J. Can. 1999, 34, 123.

11. Spengler, P.; Körner, W.; Metzger, J. W.; Environ. Toxicol. Chem. 2001, $20,2133$.

12. U.S. EPA - United States Environmental Protection Agency; Report $N^{\circ}$. EPA/630/R-96/012, Washington, DC, 1997.

13. Council, N. R.; Washington, DC: National Research Council, 1999.

14. Schäfer, A. I.; Waite, T. D.; IWA Specialist Group on assessment and control of hazardous substances in water (ACHSW), 2002.

15. Aerni, H. R.; Kobler, B.; Rutishauser, B. V.; Wettstein, F. E.; Fischer, R.; Giger, W.; Hungerbühler, A.; Marazuela, M. D.; Peter, A.; Schönenberger, R.; Vögeli, A. C.; Suter, M. J. F.; Eggen, R. I. L.; Anal. Bioanal. Chem. 2004, 378, 688.

16. Sodré, F. F.; Montagner, C. C.; Locatelli, M. A. F.; Jardim, W. F.; J. Braz. Soc. Ecotoxicol. 2007, 2, 187.

17. Liu, R.; Zhou, J. L.; Wilding, A.; J. Chromatogr., A 2004, 1022, 179.

18. IBGE - Instituto Brasileiro de Geografia e Estatística; Pesquisa Nacional de Saneamento Básico 2000, Rio de Janeiro, 2002.

19. Sodré, F. F.; Locatelli, M. A. F.; Jardim, W. F.; Water, Air, Soil Pollut. 2010, 206, 57.

20. Westerhoff, P.; Yoon, Y.; Snyder, S.; Wert, E.; Environ. Sci. Technol. 2005, 39, 6649 .

21. Dodd, M. C.; Buffle, M. O.; von Gunten, U.; Environ. Sci. Technol. 2006, 40, 1969.
22. Rosenfeldt, E. J.; Chen, P. J.; Kullman, S.; Linden, K. G.; Sci. Total Environ. 2007, 377, 105.

23. Ikehata, K.; El-Din, M. G.; Snyder, S. A.; Ozone: Sci. Eng. 2008, 30, 21.

24. Quinlivan, P. A.; Li, L.; Knappe., D. R. U.; Water Res. 2005, 39, 1663.

25. Zhang, Y.; Zhou, J. L.; Water Res. 2005, 39, 3991.

26. Fukuhara, T.; Iwasaki, S.; Kawashima, M.; Shinohara, O.; Abe, I.; Water Res. 2006, 40, 241.

27. Fernandes, A. N.; Tese de Doutorado, Universidade Federal de Santa Catarina, Brasil, 2007.

28. Fernandes, A. N.; Almeida, C. A. P.; Menezes, C. T. B.; Debacher, N. A.; Sierra, M. M. D.; J. Hazard. Mater. 2007, 144, 412.

29. Petroni, S. L. G.; Pires, M. A. F.; Munita, C. S.; Quim. Nova 2000, 23, 477.

30. Lamim, A. P. B.; Jordão, C. P.; Pereira, J. L.; Bellato, C. R.; Quim. Nova 2001, 24, 18.

31. Falone, S. Z.; Vieira, E. M.; Quim. Nova 2004, 27, 849.

32. Carvalho, P. H. V.; Jesus, A. M. D.; Prata, V. M.; Bezerra, D. S. S.; Romão, L. P. C.; Navickienne, S.; J. Braz. Chem. Soc. 2010, 21, 659.

33. Rossner, A.; Snyder, S. A.; Knappe, D. R. U.; Water Res. 2009, 43, 3787.

34. Brown, P. A.; Gill, S. A.; Allen, S. J.; Water Res. 2000, 34, 3907.

35. U.S. EPA - United States Environmental Protection Agency; Method 3535A: Solid-phase extraction, 2007.

36. Fernandes, A. N.; Giovanela, M.; Esteves, V. I.; Sierra, M. M. D.; J. Mol. Struct. 2010, 971, 33.

37. von Post, L.; Com. Soil Sci. 1924, 22, 287.

38. IPT - Instituto de Pesquisas Tecnológicas do Estado de São Paulo. São Paulo: Relatório 12, 1979.

39. INMETRO - Instituto Nacional de Metrologia, Normalização e Qualidade Industrial; Orientações sobre Validação de Métodos de Ensaios Químicos, DOQ-CGCRE-008, 2003.

40. Ribani, M.; Bottoli, C. B. G.;Collins, C. H.; Jardim, I. C. F.; Melo, L. F. C.; Quim. Nova 2004, 27, 771.

41. Changa, H. S.; Choo, K. H.; Leeb, B.; Choi, S. J.; J. Hazard. Mater. 2009, 172, 1 .

42. Gatidou, G. N.; Thomaidis, S.; Stasinakis, A. S.; Lekkas, T. D.; J. Chromatogr., A 2007, 1138, 32.

43. Labadie, P.; Budzinski, H.; Anal. Bioanal. Chem. 2005, 381, 1199.

44. Liu, Y.; Guan, Y.; Tam, N. F. Y.; Mizuno, T.; Tsuno, H.; Zhu, W.; Water, Air, Soil Pollut. 2010, 209, 333.

45. Lopes, L. G.; Marchi, M. R. R.; Souza, J. B. G.; Moura, J. A.; Lorenzon, C. S.; Cruz, C.; Amaral, L. A.; Quim. Nova 2010, 33, 639.

46. Atkins, P. W.; Physical Chemistry, $5^{\text {th }}$ ed., Oxford University Press: New York, 1994.

47. Chen, X.; Hu, J.; Water, Air, Soil Pollut. 2010, 206, 251.

48. Pan, B.; Sun, K.; Xing, B.; J. Soils Sediments 2010, 10, 838.

49. Yoon, Y.; Westerhoff, P.; Snyder, S.; Esparza, M.; Environ. Sci. Technol. 2003, 37, 3530.

50. Pan, B.; Lin, D.; Mashayekhi, H.; Xing, B.; Environ. Sci. Technol. 2008, 42,5480 .

51. Silva, L. R.; Ferreira, M. M. C.; Quim. Nova 2003, 26, 312.

52. Doğan, M.; Alkan, M.; Demirbas, Ö.; Özdemir, Y.; Özmetin, C.; Chem. Eng. J. 2006, 124, 89 .

53. Chang, Y.; Lv, X.; Zha,F.; Lei, Z.; J. Hazard. Mater. 2009, $168,826$.

54. Langmuir, I.; J. Am. Chem. Soc. 1916, 38, 2221.

55. Freundlich, H.; Z. Phys. Chem. A 1906, 57, 385.

56. Rodrigues, L. A.; Silva, M. L. C. P.; Quim. Nova 2009, 32, 1206.

57. Eren, E.; J. Hazard. Mater. 2009, 162, 1355.

58. Song, K. Y.; Parka, P. K.; Kima, J. H.; Lee, C. H.; Lee, S.; Desalination 2009, 237, 392.

59. Lagergren, S. K.; Kung. Sven. Vetenskapsakad. Handl 1898, 24, 1.

60. Weber, W. J.; Morris, J. C.; J. Sanit. Eng. Div. Am. Soc. Civ. Eng. 1963, $89,31$.

61. Almeida, C. A. P.; dos Santos, A.; Jaerger, S.; Debacher, N. A.; Hankins, N. P.; Desalination 2010, 264, 181.

62. Almeida, C. A. P.; Debacher, N. A.; Downs, A. J.; Cottet, L.; Mello, C. A. D.; J. Colloid Interface Sci. 2009, 332, 46. 